\title{
On Risk Acceptance in the Industrial Society
}

\author{
Francis Stoessel ${ }^{\star}$
}

\begin{abstract}
The public, and society in general, is concerned by risks arising from technological projects and industrial activities. Responsible handling can only be achieved if the required knowledge about the hazards, and their consequences, is available in the industry and can be communicated to the public. Chemical industries occupy a special position in this context, since accidents, even if seldom, may have a strong impact on the public.

One possible way to achieve acceptance is education and information, which facilitate communication between the different parties. Some experiences in the education of professionals and risk communication in the chemical process industry will be presented.
\end{abstract}

Keywords: Chemical processes · Communication · Education · Risk acceptance $\cdot$ Training

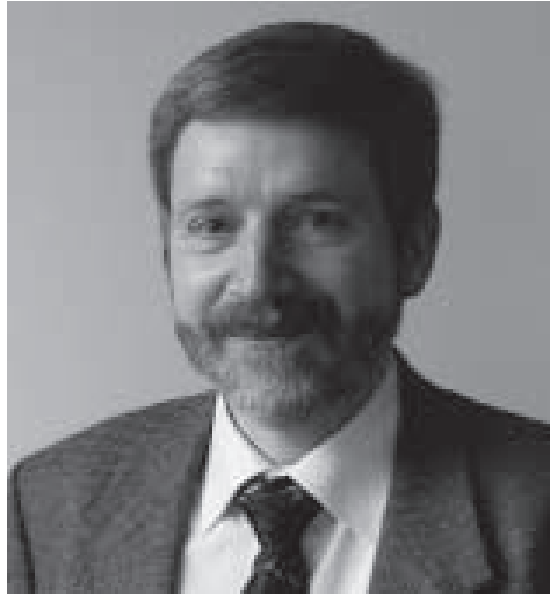

${ }^{{ }^{*} \text { Correspondence: Prof. Dr. F. Stoessel }}$ Ecole Polytechnique Fédérale de Lausanne Faculté des Sciences de Base

Institut de Sciences des Procédés chimiques et biologiques

$\mathrm{CH}-1015$ Lausanne

Tel.: +4121693 3671

Fax: +41216933190

E-Mail: francis.stoessel@epfl.ch

and

Swiss Institute for the Promotion of Safety \& Security WKL-32.3.22

$\mathrm{CH}-4002$ Basel

Tel.: +4161696 2456

Fax: +41616967072

E-Mail: fstoessel@swissi.ch

The present paper is a revised version of a communication presented at the International Conference on Safety and Risk Engineering, Malta 2001

\section{Introduction}

In the 20th century there was an evolution of science from the pure aim to understand our world towards technology and the ambition to modify it. 'Modern science is at the junction between technique and theory, the systematic alliance between the ambition to mould the world and this to understand it' [1]. Technological progress confers new powers on humankind, but also means creation of new risks. Science brings liberation and progress, but simultaneously it is a source of the greatest threats. Current technology renders possible things that some years ago would have been an abstract dream. The question now arises: must something be done simply because it is feasible? Is it advisable to do so? Is it advantageous? If yes, for whom? [2]. If understanding the world cannot be contested, modifying our environment is subject to justified discussion.

Basically, every technological project has at least two objectives: certainly one of them is to improve the quality of life while the other is of an economic nature and aims to generate profit. By improving the quality of life we mean, for example, to build comfortable places to live, to have good means of transportation, to improve health, to have comfortable clothing etc. The profit can be of a financial nature, but also of a more abstract nature, such as recognition, satisfaction, etc. As every medal has two sides, these activities may also generate some negative consequences, such as environmental impact and occasional incidents. Therefore a balance has to be established between the benefits and the price society or individuals have to pay. This balance is a potential source of conflict since the opinions will be very different, depending on who is considering the problem. There is a great temptation to reap immediate benefits, but this must be balanced by the benefits future generations will be able to earn or not, hence the balance must be established on a long-term time scale. For this reason it is also imperative to establish a broad awareness of the benefits and of the risks related to new technologies or to technological projects in general.

This will be the subject of the first section describing the framework of risk communication and establishing some conditions for an efficient debate. In the second section, some examples stemming from the chemical process industry, which is strongly implicated in such discussions, will be presented. Finally, some of the options available when teaching about risks will be discussed. 


\section{Risk Acceptance}

The actors involved in the debate about the risks linked to technological projects are very different by nature. For this reason mutual understanding is vital but difficult to achieve. Here different attitudes in the face of risk will be discussed (Fig. 1). This allows the problems arising from risk communication to be identified and also for some conditions required for risk acceptance to be defined.

\subsection{Human Attitudes when Facing Risks}

Beside this purely operative attitude, it is worth looking at different kinds of risks by deriving various human attitudes from those found in the Greek philosophy:

In face of huge human projects (pharaonic projects) that are often considered to be beyond measure (hubris, $v \beta \rho \imath \sigma)$, a general adhesion or even enthusiasm can be achieved. This is true as long as things go well, but in case of a catastrophe, those responsible will be sought out and they will have to account for the consequences.

In face of risks stemming from nature such as earthquakes, storms and so forth (anagkê, $\alpha v \alpha \gamma \kappa \eta$ ), the attitude will be fatalism. These risks cannot be avoided; they impose even on the gods, so there is no escape or surrender.

In face of spontaneous or natural causes (automatos, $\alpha v \tau \sigma \mu \alpha \tau o \sigma$ ) humans will fight and try to act against them. Technology developed in recent years allows a shift from surrender towards combat, thus preventing such catastrophes.

In face of risks relying on chance (tuchê, $\tau v \chi \eta$ ), the common human attitude is to seize the chance offered or even to cheat in order to 'help' chance.

If it is generally recognized that humans need safety, at the same time they are striving for risks. This is not a paradox since the driving force is to recover safety after having taken risks. It is a dynamic situation (Flow-Erlebnis) with a constant change between safety and risk [3]. A task without any surprises, without challenges, is a boring task; the pleasure linked to work stems from the resolution of problems. The opportunity to take risks arises because problem solving is often an innovation, i.e. a step towards the unknown and, once the solution has been found, safety is recovered leaving a feeling of satisfaction. But this image cannot be pushed to the extreme, i.e. an

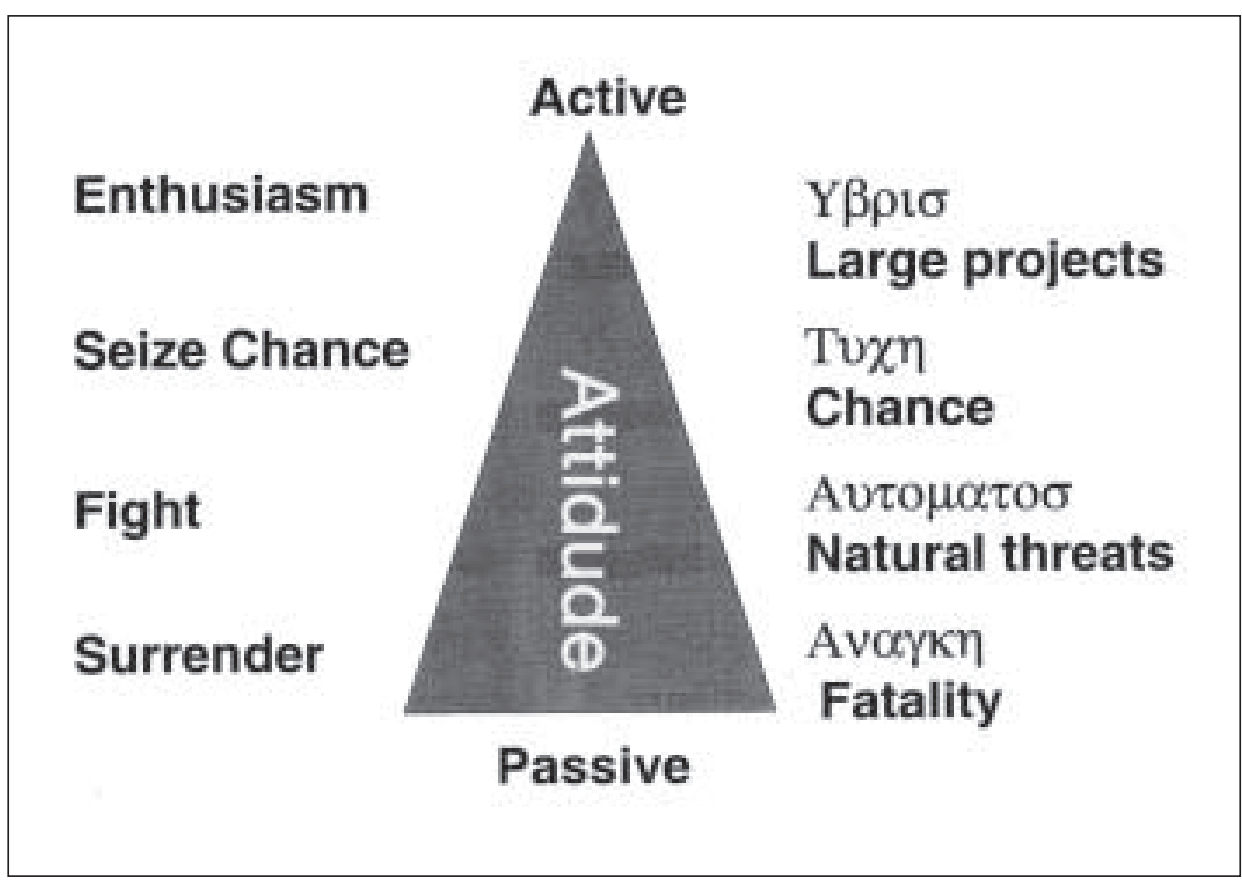

Fig. 1. Human attitudes may be very diverse depending on the context and on the nature of the risk.

employee lacking in security, or who is always subject to pressure, is unable to be innovative. Creativity and innovation require a certain degree of safety. At the other extreme a pronounced lack of safety may cause humans to look for refuge in faith, sects or even esoteric beliefs.

Often risk evaluation is performed in everyday life in a more or less conscious way: for example in sporting activities, or by driving fast etc. Sometimes high risks are accepted collectively like populations establishing themselves in dangerous environments like in the vicinity of a volcano or in a valley subject to flooding (Nile Valley) in order to profit from the higher soil fertility. Another example is learning by trial and error, where risk becomes a learning factor. Therefore it is generally admitted that one cannot learn without taking risks.

In the technological context, the most familiar attitude for engineers stems from the classical definition of risk used by industries and insurance companies: 'Risk is a combination between the severity and the probability of occurrence of an incident'. This combination is often expressed as the product between severity and probability of occurrence. It is essentially used to evaluate risks qualitatively or quantitatively using different scales. In the fine chemicals industry, a simple three level scale (high, medium, low) is often used for this purpose. There are also more sophisticated scales using a more rigorous quantification of the risks, sometimes imposed by law. The fact that the concept of risk deals with a potential incident implies that such incident scenarios have been previously identified, which in turn implies that a risk analysis has been performed. The risk evaluation is the result of a systematic analysis of failures or deviations from normal operating conditions and aims to facilitate the choice and help in the design of riskreducing measures. This is a purely operative attitude in the face of risk.

Attitudes in the face of risk may fundamentally differ but there is an additional difference, namely in the interest the different parties may have.

\subsection{Problems Arising from Risk Communication}

Since communication is driven by objectives, it cannot be neutral and often leads to a conflicting situation. The problems linked to risk communication have different roots:

Firstly there is often no congruence between those who profit and those who will pay the price for it (Fig. 2). This is a political problem since it addresses the decision-making process in a society. It will only be mentioned in passing here.

The second lies in the educational background of the parties involved and plays an important role. In fact there is a gap between the know-how of professionals, safety or environment experts, 
Fig. 2. For industrial projects, often there is no congruence between those who benefit and those who are exposed to drawbacks. and the public. Some ways to close the gap between experts and professionals will be shown, using the chemical process industry as an example.

The viewpoint of project owners is different from that of the general population. Project owners are interested in profit and their freedom must be governed or even limited by responsibility. The point of view of the population, those who will be subject to the consequences, is defined by human rights, looking after oneself well, and by regarding the project owner as the guilty party when safety fails. For this reason, a counter power is required. It may consist of independent commissions to cover the political aspects or ethical commissions to cover the industrial aspects. Without such a counter power risk may be hazardous to the population. This is a problem that must be solved at the political level, but in the sense of the French 'Le politique', meaning improvement of civic life and not 'La politique' meaning striving for power.

In discussions about risks, each party acts with reference to its own system of values. But the same object may have different values for different persons. Since ethics is based on a system of values that attempts to guide actions and behaviours from possible evil to good, there is a strong link between ethics and risk. In this context, we must be conscious of the plurality of ethics, which should not be considered as laws or morals, but rather as a consensual horizon towards which we have to work [4]. The distinction between the ethics of responsibility, which are linked to the political, juridical and

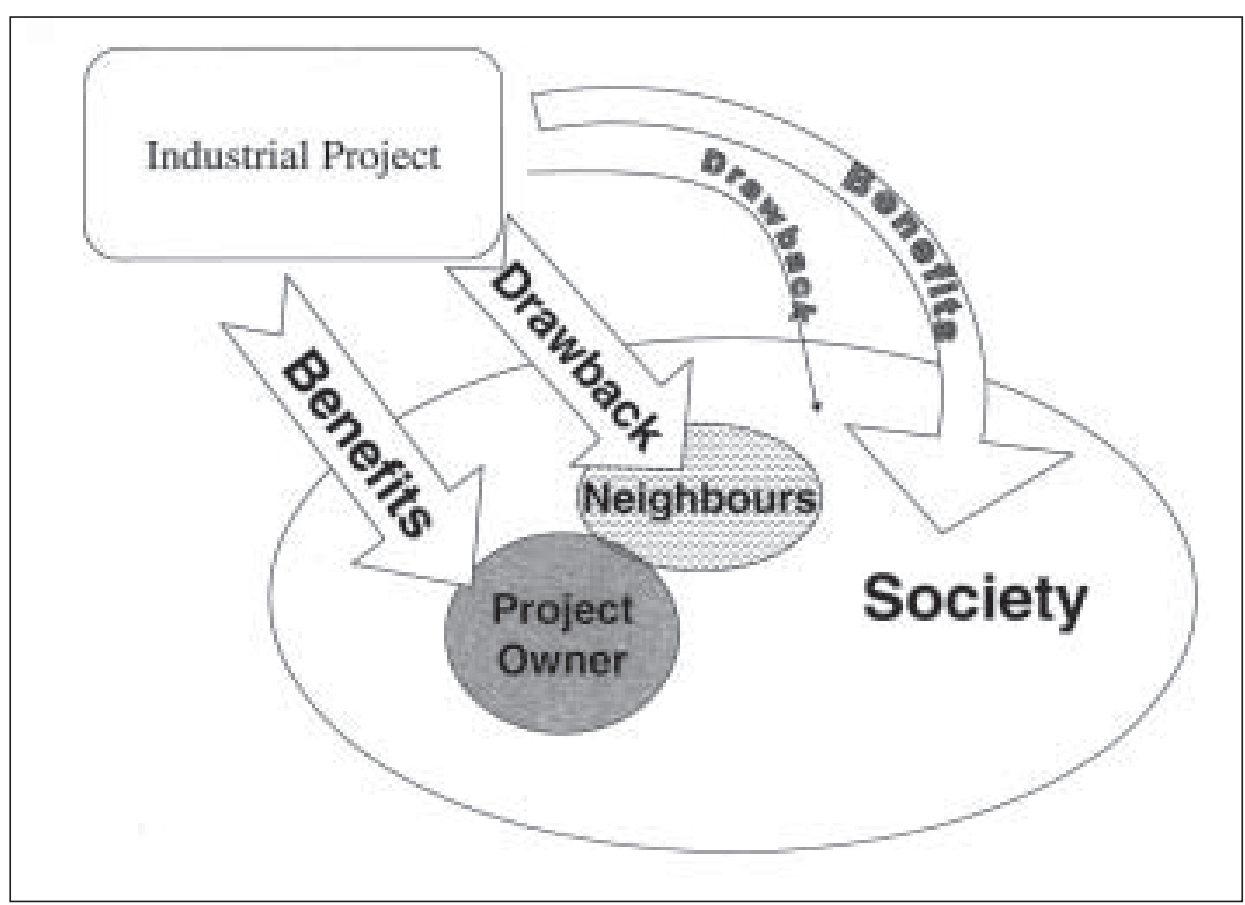

economic fields and conviction ethics, which have their roots in purely personal opinions, is important.

Scientists are often considered as cold technocrats who lack or have only a poor conscience, whereas humanists are threatened by technology. The roots of this situation must be sought in the too strict separation of the sciences during the education process.

\subsection{Conditions for Risk Acceptance}

There may be many different actors involved in technological projects. For our purposes we can identify two categories: the project owner (industry) and the public (which may be the future user or only a neighbour of the project). If we consider the public, the prerequisite for risk acceptance is to have an insight into the project. This can only be achieved if one knows what the project consists of exactly, how it works, what the benefits will be and what the drawbacks are. Then the fundamentals for the appreciation of the related risks are established, which in turn allows decisions to be made about the necessity of a project or its rejection, founded on a sound basis. This problem has the same ethical background as therapeutic decision-making by physicians. Know-how and know-how transfer between professionals and the public are particularly important in this context.

This implies a dialog between the different parties, thus reciprocal confidence is essential to achieve acceptance. This is certainly the most ambitious objective in this framework. An example stemming from the French nuclear industry can be quoted here, where engineers established their houses in the immediate neighbourhood of nuclear plants in order to demonstrate their own confidence.

Accepting risk also implies that a choice between different options for the project is possible. This is a requirement if acceptance should be the result of negotiations. Here some basic rules should never be forgotten: Humans are free and responsible. Humans take risks only because they use what has been given to them: to dominate and serve nature. This also means that they will have to account for their acts.

Finally accepting risks implies a counterpart, which may consist of different elements ranging from the responsibility of the project owners, who must provide protection from potential catastrophes by every possible reasonable means, to a guarantee of compensation in case of damage.

The public will accept what it understands and what it feels to be necessary.

Thus education of the public as well as of professionals is required to facilitate the risk discussion. Some examples of education or communication stemming from the chemical process industry will be given in the next section.

\section{Examples from the Chemical Process Industry}

Projects in the chemical process industries are of increasing complexity, and often use diversified and innovative technologies. This has two consequences:

The public can barely understand what the processes really are and what the impact may be. 
Multi-disciplinarity becomes quite common, which implies a new communication problem between professionals of different disciplines.

These aspects will be covered in the next subsections.

\subsection{Communication with the Public}

The public has a relatively good perception of civil engineering projects. They are visible and the function can be understood by practically everybody. This is quite different in the chemical process industries where the necessity is not directly comprehensible for the general public. Thus a further effort in explaining what is done in a chemical plant is required. This can be achieved by transparency, trying to become a good neighbour, but it remains a demanding task particularly since the average level of education of the public is inadequate.

After the Sandoz fire in November 1986 , there was a crisis in the city of Basel, mainly due to the loss of confidence of the citizens in 'their' chemical industry. Never before had an accident been perceived as such a threat. There were neither fatalities nor injuries, but extensive water pollution of the Rhine river and air pollution over the town. To deal with this, Ciba-Geigy started a information campaign for the public, starting with the immediate neighbours of the plant. This campaign consisted of different actions and followed the principles of transparency, credibility, and trace ability [5].

The neighbours were informed about activities in the plant by a journal issued quarterly. This journal contained articles about safety in the framework of new projects, existing production plants and research activities. The location, nature and amount of dangerous material stored in the plant area was made public. This was accompanied by the program 'Glassy Plant': neighbours were invited for visits 'à la carte', i.e. they could choose what they wanted to see and when they wanted to be invited for a visit in small groups. Some chemists had the responsibility of informing the neighbourhood schools in the case of an alarm. The schools were equipped with printers that allowed information about the nature of the alarm and the appropriate behaviour for the situation to be transmitted. Such alarms were simulated to enable training of teachers and children.

These actions were completed by a series of exercises with simulated incidents and involving the industry, authorities and media of three countries (Swit- zerland, Germany and France). These exercises focused on communication problems during an emergency situation and were of great help in establishing direct and personal contacts in addition to creating familiarity with such situations.

This transparent way of disseminating information is not the easiest by any means. Regaining the confidence of the population in their chemical neighbour takes a long time, and this effort never ceases. But in a long-term perspective, an open information policy is certainly the best way to go, because the population is allowed to form its own opinion about the plant and its safety management.

\subsection{Communication between Professionals}

The educational background of the professionals involved in projects is often so different that communication between engineers may become difficult. This problem is reinforced when experts are required to work in project teams. In the case of chemical process safety, especially when chemical reactions are involved, an additional difficulty arises: the phenomena considered, for example in runaway reactions or thermal explosions, are strongly non-linear, which rends risk evaluation particularly tricky. Often the behaviour of a reactor is difficult to comprehend, which makes the predictions required in risk analysis particularly tough.

This has been experienced in the fine chemical industries where all the incidents which occurred in the recent past could be thoroughly understood, and more critically, could have been avoided if the knowledge required for their explanation had been used during the process design. Here we face another gap between the know-how of experts and the know-how of professionals, i.e. the know-how is available but not at the right place. Here the challenge is for the experts who must develop ways of communication to ensure a good understanding of their know-how by professionals in different specialties. This problem can be solved, at least to a certain extent, by training and by the development of a common language platform for communication.

Education curricula in chemistry only very occasionally include lectures about process safety. This lack is being corrected in an increasing number of universities and engineering schools. In order to fill this gap special training seminars and courses have been organized within the industries. The larger companies opened these courses to chemists and engineers from small and medium companies and to members of the authorities, who may not have the required background to be able to assess the safety of chemical processes. This open policy in training was essentially driven by a sense of responsibility towards the public.

These courses comprise a basic course on process safety aiming to give a general background and also to familiarize the participants with risk analysis techniques. More specialized courses have focused on explosion protection, electrostatics, thermal process safety, and industrial hygiene. These courses have a strongly interactive basis, meaning that each module is accompanied by case studies, based on real cases, which are tackled in small groups. This is to allow for maximum efficiency in the knowhow transfer. Participation in these courses was mandatory for every engineer or chemist in charge of a plant unit or in process development units. These courses still exist and are delivered by an outsourced company (The Institute for Promotion of Safety and Security) stemming from the largest chemical companies in Basel.

If the newly acquired knowledge is not used regularly in time, memory fails and the efficiency is lost. This is often the case for production chemists who perform risk assessments only occasionally. For these cases it is essential to have access to a tool which can refresh the memory. Computer programs can fulfil this role in a very efficient way: they can be loaded on a personal computer and are accessible at all times. With an interactive program, the user can check his performance by answering questions, he can progress at his own pace, jump back if required etc. In the following, some examples of interactive computer tools well adapted or especially designed for training chemists in the field of thermal process safety are presented [6].

\section{Teaching Risk Assessment}

\subsection{Computer-aided Tutoring: (Thermal Safety Tutorial, Electrostatics Tutorial)}

The user is guided through a series of different case studies where he learns the essential aspects of process safety: data evaluation, interpretation, risk assessment and planning risk reduction measures. In a help file structured as hypertext, the user can navigate his way through the different basic concepts [7]. 


\subsection{Knowledge-based Tools: (Thermal Safety Assistant)}

This tool allows real problems related to the assessment of thermal risks of industrial processes to be solved. It is based on the principle of learning by doing. It forces the systematic collection of data, helps in interpretation, and explains the consequences of malfunction during the industrial operation. The help file, structured as hypertext, allows the basic concepts to be learnt or the verification of understanding. Additionally, a database (heat capacities, reaction enthalpies...) and a collection of calculation programs enabling, for example, the heat capacity of a mixture or the energy of activation of a reaction to be calculated has been incorporated.

\subsection{Simulation-based Tools}

In chemical reaction engineering and therefore also in the assessment of the safety of chemical reactions, one deals with strongly non-linear systems. This non-linearity stems essentially from the Arrhenius Law, describing the effect of temperature on the reaction rate. But the human brain has some difficulties in thinking in a non-linear fashion. Thus in the education of chemists it is important to give them a feeling for these relationships and to help them to understand the effect of variation of parameters on the behaviour of a reacting system. Using a dynamic simulation program greatly helps to achieve this. It gives also access to situations which cannot be achieved experimentally. As an example, runaway curves can easily be calculated by simulation, whereas the systematic experimental exploration of such phenomena cannot be carried out for obvious reasons. The effect of scale-up on a heat balance can easily be studied by simulation, using for example the sensitivity analysis technique. So the simulation technique allows cheap 'calculation experiments' to be multiplied, which is a great help, if not the only way, in understanding the behaviour of complex and non-linear systems. For this reason it is of great help in teaching chemists as well as undergraduates.

\section{Discussion and Conclusions}

The key to a successful discussion about risks, perhaps leading to acceptance, is information and education. This is a prerequisite, but there is also a danger in the teaching purpose. It must be undertaken by regarding the other persons as partners (sharing power) rather than leading them to unfamiliar cultures. This would be equivalent to gaining domination over others, like for instance factory owners in the 19th century providing workers' children with schooling in order to keep the workers under control. So the title of the paper could have been 'Can we teach people to accept risk?' and the question is to know who will do the teaching and how. It is the responsibility of the companies or project owners to inform in an open way.

The society problems arising from risk acceptance cannot be solved solely by modification of educational programs, but this could certainly help to improve the situation to a great extent. Here we mean lack in education of the public and of training of professionals, both in technical aspects as well as society and ethical aspects. Emerging New Learning Technologies allow tools to be created for the education of the public or for the training of professionals. Some examples stemming from experience in the fine chemical industry have been shown.

It is the responsibility of society to provide people with a minimum level of knowledge, which allows a balance to be made between benefits and risks of projects on a rational basis. Thus basic education programs should introduce a cost benefit analysis and give an ethical background in addition to the technical background.

This must also be implemented on a long-term scale: The decisions we make today define what humanity will be in future. In this perspective we must also remember that long-term survival of an industrial society requires the aptitude for global competition, the integration in the society (acceptation) and a natural environment [8]. The immediate benefits must be balanced by the benefits for future generations.

\section{Acknowledgment}

The author wishes to thank to Dr. Michel Cordier for his constructive and valuable suggestions during the preparation of the manuscript and his critical review of the paper. The author is also grateful for financial support by the Swiss Institute for the Promotion of Safety \& Security.

Received: February 19, 2002

[1] I. Prigogine, I. Stengers, 'La nouvelle alliance, métamorphose de la science', Gallimard, 1979.

[2] G. Bourgeault, 'L'éthique et le droit face aux nouvelles technologies biomédicales', De Boeck Université, Bruxelles, 1990.
[3] F. Von Cube, 'Gefährliche Sicherheit in Leben mit Risiko', Chr. Merian Verlag Basel 1993.

[4] D. Müller, 'Les éthiques de responsabilité dans un monde fragile', Fides, 1998.

[5] J.R. Randegger, 'Die chemische industrie im Umgang mit Risiken in Leben mit Risiko', Chr. Merian Verlag, Basel, 1993.

[6] F. Stoessel, H. Fierz, P. Lerena, G. Killé, 'Recent developments in the assessment of thermal risks of chemical processes', Organic Process Research and Development, vol. 1, no. 6, 1997, pp 428-434.

[7] The program is available from the Expert Commission for Safety in the Swiss Chemical Industry (SUVA Section Chemistry, CH 6000 Lucerne, Switzerland).

[8] K. Hungerbühler, J. Ranke, T. Mettier, 'ChemischeProdukte und Prozesses, Grundkonzepte zum umweltorientierten Design', Springer Verlag, Berlin, 1998. 